\title{
¿HA AUMENTADO LA EFICIENCIA DE LA POLÍTICA MONETARIA EN MÉXICO?
}

\author{
Stephen G. Cecchetti \\ Alfonso Flores Lagunes \\ Stefan Krause ${ }^{1}$
}

Marzo de 2001

Documento de Investigación No. 2001-01

Dirección General de Investigación Económica

BANCO DE MÉXICO

\footnotetext{
${ }^{1}$ Cecchetti: The Ohio State University y NBER; Flores Lagunes: The Ohio State University; Krause: The Ohio State University. Las opiniones contenidas en este documento representan las de los autores y no necesariamente las del Banco de México. Los autores agradecen a Miguel Messmacher y Alejandro Werner por sus comentarios a una versión preliminar del documento, a los participantes en el Programa de Presentaciones de la Dirección de Estudios Económicos del Banco de México, y a Daniel Garcés Díaz por compartir su base de datos de la economía mexicana. A. Flores Lagunes agradece la hospitalidad de la Dirección de Estudios Económicos del Banco de México durante su estancia en el verano de 2000.
} 


\title{
¿HA AUMENTADO LA EFICIENCIA DE LA POLÍTICA MONETARIA EN MÉXICO?
}

\author{
Stephen G. Cecchetti \\ Alfonso Flores Lagunes \\ Stefan Krause
}

Marzo de 2001

Documento de Investigación No. 2001-01

\section{RESUMEN}

México ha experimentado una menor variabilidad en la tasa de inflación y en la producción industrial en la década de los noventa, en comparación con la década anterior. La presente investigación examina directamente el papel que ha jugado la política monetaria en la consecusión de esta mayor estabilidad macroeconómica.

El análisis incluye la estimación de la frontera de eficiencia que resulta del trade-off que enfrenta la autoridad monetaria entre la variabilidad de la inflación y la del producto. Esta frontera se obtiene para dos subperiodos y se utiliza en la elaboración de medidas de ganancia (o pérdida) de eficiencia en el manejo de política monetaria.

Nuestros resultados indican que la eficiencia de la política monetaria en México ha aumentado en los últimos años. Además, su contribución relativa para alcanzar una mayor estabilidad macroeconómica es de $47 \%$ o $93 \%$, dependiendo de los supuestos que se hagan acerca de los objetivos de política económica del Banco de México. 


\section{¿HA AUMENTADO LA EFICIENCIA DE LA POLÍTICA MONETARIA EN MÉXICO?}

\section{Introducción}

Durante los últimos años un número de países ha experimentado una disminución en las variabilidades observadas tanto de la inflación como del producto. ${ }^{2}$ En el caso particular de México, la varianza ${ }^{3}$ de la inflación se redujo de $7.02 \%$ entre 1982 y 1988 a $1.76 \%$ entre 1991 y 1997, mientras que, para los mismos periodos, la varianza del logaritmo natural de la producción industrial disminuyó de $0.40 \%$ a $0.19 \%$.

Este incremento en la estabilidad macroeconómica ha sido resultado de la combinación de dos factores ${ }^{4}$ : 1) una caída en la varianza de las perturbaciones de oferta (ver adelante); y 2) un incremento en la eficiencia de la política monetaria en México. El objetivo de esta investigación consiste en tratar de identificar la contribución relativa de cada uno de estos factores.

El desempeño de la política monetaria se puede evaluar utilizando el concepto de trade-off entre la variabilidad de la inflación y la del producto que la autoridad monetaria enfrenta. De manera general, se considera una economía que enfrenta dos tipos de perturbaciones, las de demanda agregada - que mueven al producto y a la inflación en la misma dirección- y las de oferta agregada - que mueven al producto y a la inflación en direcciones opuestas. Dado que la política monetaria puede mover al producto y a la inflación en la misma dirección, ésta puede contrarrestar completamente las perturbaciones de demanda, mientras que las perturbaciones de oferta obligarán a la autoridad monetaria a decidir entre aceptar una mayor variabilidad en la inflación o en el producto.

Como resultado de dicho trade-off es posible construir una frontera de eficiencia para la política monetaria, en la cual se minimizan las variabilidades de inflación y producto (ver

\footnotetext{
${ }^{2}$ Ver Cecchetti y Ehrmann (1999), entre otros.

${ }^{3}$ Medida como la dispersión relativa a la media aritmética.

${ }^{4}$ En este documento sólo se contemplan los efectos de la política monetaria sobre la estabilidad macroeconómica, dado que los efectos (estabilizadores o desestabilizadores) de la política fiscal se incluyen dentro de las perturbaciones de demanda agregada (ver Sección II).
} 
Gráfica 1). ${ }^{5}$ Si la política monetaria es óptima, la autoridad monetaria seleccionará un punto sobre dicha curva, el cual dependerá de las preferencias que tenga con respecto a las variabilidades de la inflación y del producto. La ubicación de la frontera de eficiencia está en función de la varianza de las perturbaciones de oferta; entre menor sea dicha varianza, más cercana al origen se encontrará la frontera. Si la política monetaria no es óptima, la eficiencia de ésta será mayor entre más cercano a la frontera se ubique el punto de desempeño de la economía.

\section{Gráfica 1: Frontera de eficiencia y punto de desempeño}

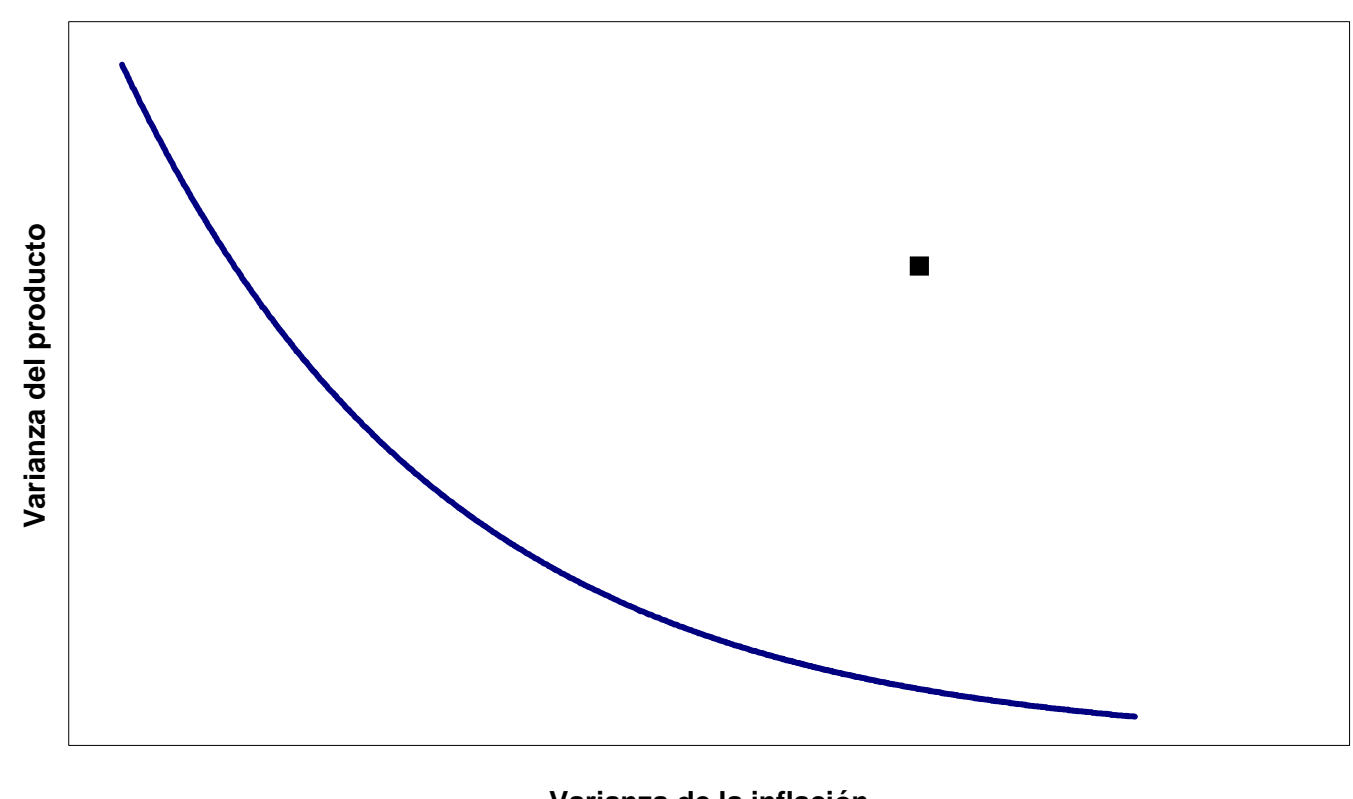

Si se considera exclusivamente la caída en las variabilidades observadas de la inflación y del producto no es posible identificar las contribuciones relativas de una política monetaria más eficiente y de una reducción en la varianza de las perturbaciones de oferta agregada. Por ello, la estrategia de este trabajo consiste en desarrollar medidas de ganancia (o pérdida) de eficiencia de la política monetaria netas del cambio en la varianza de las perturbaciones de oferta agregada, para lo cual se requiere comparar el punto de desempeño con la frontera de eficiencia para dos subperiodos distintos.

El presente documento se encuentra organizado de la siguiente manera: en la Sección II se presenta y estima un modelo estructural para la economía mexicana, en la Sección III se muestra la forma de obtener la frontera de eficiencia a partir del modelo estructural, mientras

\footnotetext{
${ }^{5}$ Esta frontera es conocida en la literatura como Curva de Taylor (Taylor, 1979).
} 
que en la Sección IV se introducen las medidas a ser utilizadas con el fin de cuantificar la ganancia o pérdida de eficiencia en el manejo de la política monetaria. En la Sección V se presentan los principales resultados y se concluye en la Sección VI.

\section{El Modelo Estructural}

Para calcular la frontera de eficiencia se requiere conocer el comportamiento dinámico de la economía bajo estudio y hacer supuestos sobre los objetivos de la política monetaria. En esta sección se procede a diseñar y estimar un modelo que se ajuste lo más cercanamente posible a la estructura de la economía mexicana.

El modelo estructural propuesto para México es uno de dos variables, muy similar al utilizado por Rudebusch y Svensson (1999) para los Estados Unidos, con la excepción de que se incluyen variables explicativas propias de una economía abierta. Este modelo lineal es manejable y permite obtener un buen ajuste empírico a los datos, tanto para la muestra total como para ambos subperiodos analizados. ${ }^{6}$ Se compone de las siguientes dos ecuaciones:

$$
\begin{aligned}
\pi_{t+1} & =\sum_{l=0}^{2} \alpha_{1 l} \pi_{t-l}+\alpha_{13} y_{t}+\alpha_{14} y_{t-1}+\alpha_{15} p x_{t}+\alpha_{16} w_{t}+\alpha_{17} c r_{t+1}+\varepsilon_{1 t+1} \\
y_{t+1} & =\sum_{l=0}^{2} \alpha_{2 l} y_{t-l}+\alpha_{23}\left(i_{t}-\pi_{t}\right)+\alpha_{24} d e v_{t}+\varepsilon_{2 t+1}
\end{aligned}
$$

La primera ecuación representa una oferta agregada o Curva de Phillips, donde se relaciona a la inflación $(\pi)$ medida en desviaciones de su media con la brecha del producto $(y)$ medida con el logaritmo de la producción industrial una vez eliminada su tendencia, y con tres rezagos de la inflación que representan la formación de expectativas inflacionarias; las otras variables explicativas son los precios externos $(p x)$ medidos por la suma de la tasa de crecimiento del tipo de cambio nominal y la inflación de los Estados Unidos; los salarios $(w)$ medidos por la tasa de crecimiento del índice de salarios; y una variable binaria $(c r)$ que captura las crisis cambiarias que experimentó la economía mexicana en 1983 y 1995. Las variables $p x$ y $w$ fueron incluidas para aumentar el poder predictivo de la ecuación de oferta agregada, siguiendo el documento de investigación de Garcés Díaz (1999). La segunda ecuación representa una demanda agregada o curva IS que relaciona la brecha del producto (y) con tres de sus propios rezagos, la tasa de interés real de corto plazo $(i-\pi)$, y la tasa de devaluación real (dev).

\footnotetext{
${ }^{6}$ Rudebusch y Svensson mencionan un número de características de este tipo de modelo y método de estimación con respecto a un par de modelos usados por la Reserva Federal de los Estados Unidos. Adicionalmente, justifican el modelo desde un punto de vista de política monetaria práctica. El lector es referido a ese documento para los detalles.
} 
Tanto $\varepsilon_{1} \operatorname{como} \varepsilon_{2}$ son errores con media cero. Estos pueden ser interpretados como combinaciones lineales de perturbaciones de oferta y demanda agregada en la economía. Suponiendo que no existe correlación entre dichas perturbaciones, $\varepsilon_{1}$ y $\varepsilon_{2}$ pueden expresarse de la siguiente forma:

$$
\begin{aligned}
& \varepsilon_{1 t+1}=\beta_{11} d_{t+1}+\beta_{12} s_{t+1} \\
& \varepsilon_{2 t+1}=\beta_{21} d_{t+1}+\beta_{22} s_{t+1}
\end{aligned}
$$

donde $d$ representa las perturbaciones de demanda y $s$ las de oferta agregada, ambas con media cero y varianza constante. Una política monetaria eficiente utilizaría la tasa nominal de interés (el instrumento de la política monetaria) con el fin de contrarrestar completamente las perturbaciones de demanda agregada. En vista de que la tasa de interés sólo aparece en la segunda ecuación del modelo estructural, $\varepsilon_{2}$ incorporaría únicamente las perturbaciones de oferta agregada (esto es, $\beta_{21}=0$ ), en caso de que la política monetaria fuese eficiente.

La estimación del modelo especificado en (1) y (2) se lleva a cabo a través de mínimos cuadrados ordinarios para cada una de las ecuaciones de manera individual, con datos de la publicación de Indicadores Económicos Principales de la OCDE y del Banco de México. ${ }^{7}$ Con el objetivo de obtener evidencia estadística para dividir la muestra completa, se aplica una de las pruebas de cambio estructural de Andrews (1993). Dicha prueba se basa en el cálculo del valor supremo de los estadísticos de la razón de máxima verosimilitud (LR) para todos los posibles puntos de cambio estructural en el $70 \%$ central de la muestra completa. Para la ecuación de inflación, dicha prueba arroja un valor del estadístico máximo de LR de 28.74 para el primer trimestre de 1988, con lo cual se rechaza la hipótesis nula de no cambio estructural en los coeficientes al 1\% de significancia estadística (valor crítico igual a 23.6); mientras que para la ecuación de la producción industrial el valor máximo de LR es de 6.05 en el cuarto trimestre de 1986, con el cual la hipótesis nula no se puede rechazar al 10\% de significancia estadística (valor crítico igual a 15.17).

Existe, por tanto, evidencia estadística para dividir la muestra en dos superiodos. ${ }^{8}$ Para efectos de estimación, se considera el primer subperiodo de 1982:I a 1988:IV y el segundo de 1991:I a 1997:IV.

\footnotetext{
${ }^{7}$ Se aplicó una prueba estadística para detectar una posible correlación contemporánea entre los errores de ambas ecuaciones. Dado que no se puede rechazar la hipótesis nula de ausencia de dicha correlación, mínimos cuadrados ordinarios es un método eficiente en este caso.

${ }^{8}$ La prueba de Andrews (1993) fue también aplicada a cada uno de los subperiodos mencionados sin poderse rechazar la hipótesis nula de no cambio estructural en los coeficientes para la ecuación de la producción industrial. Para la ecuación de la inflación la hipótesis nula de no cambio estructural en los coeficientes se rechaza para el primer subperiodo; no obstante, hay que tomar en cuenta los pocos grados de libertad con que se cuenta al estimar los 8 parámetros de dicha ecuación con cada una de las submuestras requeridas por la prueba.
} 
La Gráfica 2 corrobora dicha subdivisión al resultar evidente que la tasa de inflación trimestral anualizada cae dramáticamente después del segundo trimestre de 1988. Esta evidencia es compatible con el cambio estructural experimentado en México a finales de la década de los ochenta, el cual incluyó medidas tales como la apertura y desregulación económica y comercial. La división de la muestra también coincide con el Pacto de Solidaridad Económica llevado a cabo en $1988 .^{9}$

\section{Gráfica 2: Inflación Trimestral Anualizada}

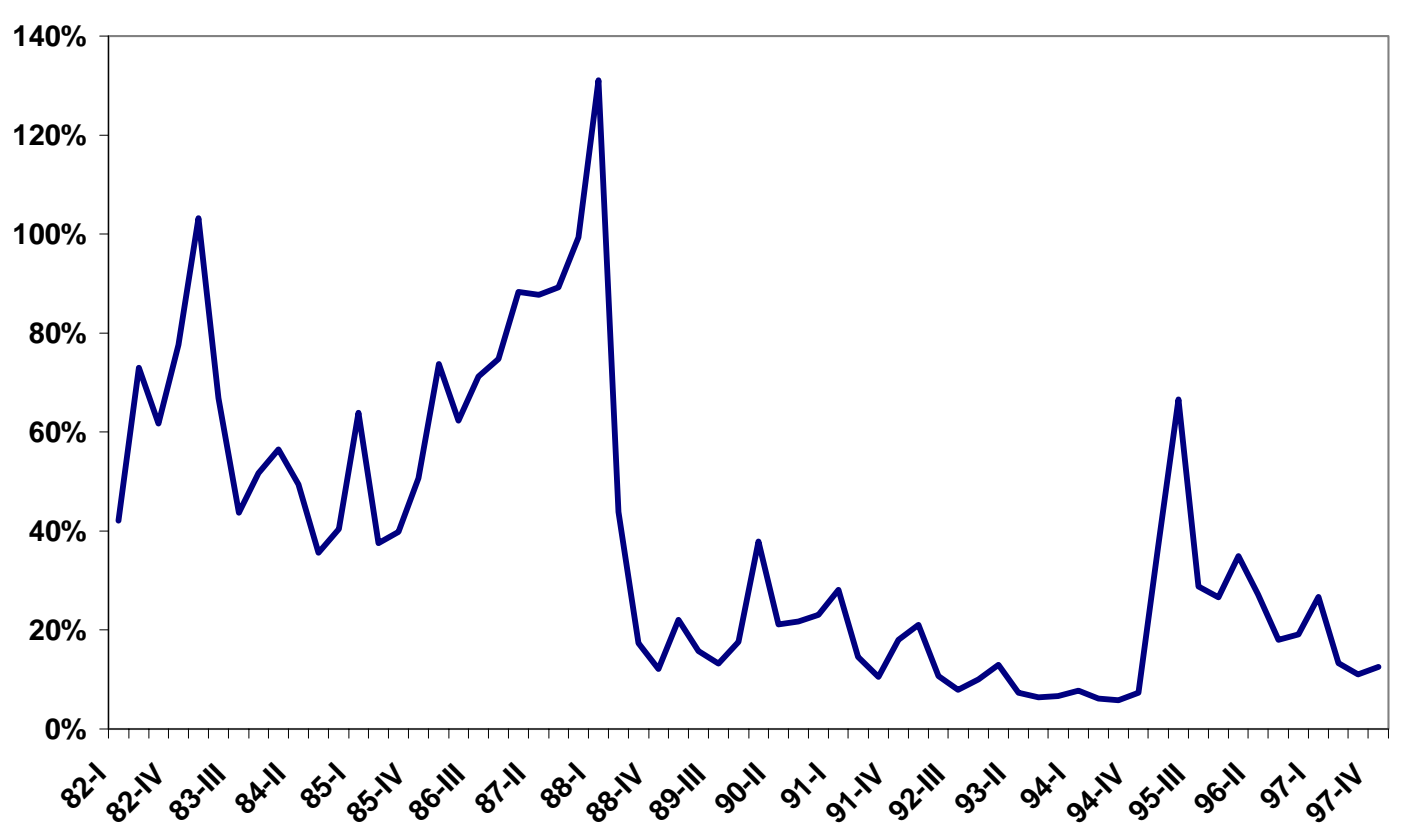

Uno de los aspectos más importantes que el modelo estructural utilizado debe satisfacer es el de rendir funciones de impulso-respuesta (FIR) apropiadas, en particular en lo que respecta al efecto de un aumento de un punto porcentual en la tasa de interés nominal sobre la inflación y el producto. La teoría indica que las gráficas de las FIR de la inflación y del producto que resultan de una perturbación de tasa de interés deben presentar una forma de "U"; esto es, ambas variables disminuyen y eventualmente tienden a regresar a su nivel inicial al ocurrir un aumento en la tasa de interés, manteniendo todo lo demás constante.

\footnotetext{
${ }^{9}$ Las ecuaciones estimadas para el periodo completo y cada uno de los subperiodos se presentan en el Cuadro A.1 en el Apéndice. Se realizaron pruebas de no cointegración para el periodo entero y los subperiodos, rechazándose la hipótesis nula en todos los casos a un nivel de significancia del 1\%. Finalmente, no se encontró evidencia estadística de autocorrelación en los errores.
} 
Como se muestra en el panel izquierdo de las Gráficas 3.1-3.3, el modelo estructural rinde FIRs aceptables tanto para la muestra completa como para ambos subperiodos. ${ }^{10}$

\section{Gráfica 3.1 Funciones de Impulso-Respuesta (Periodo Completo)}
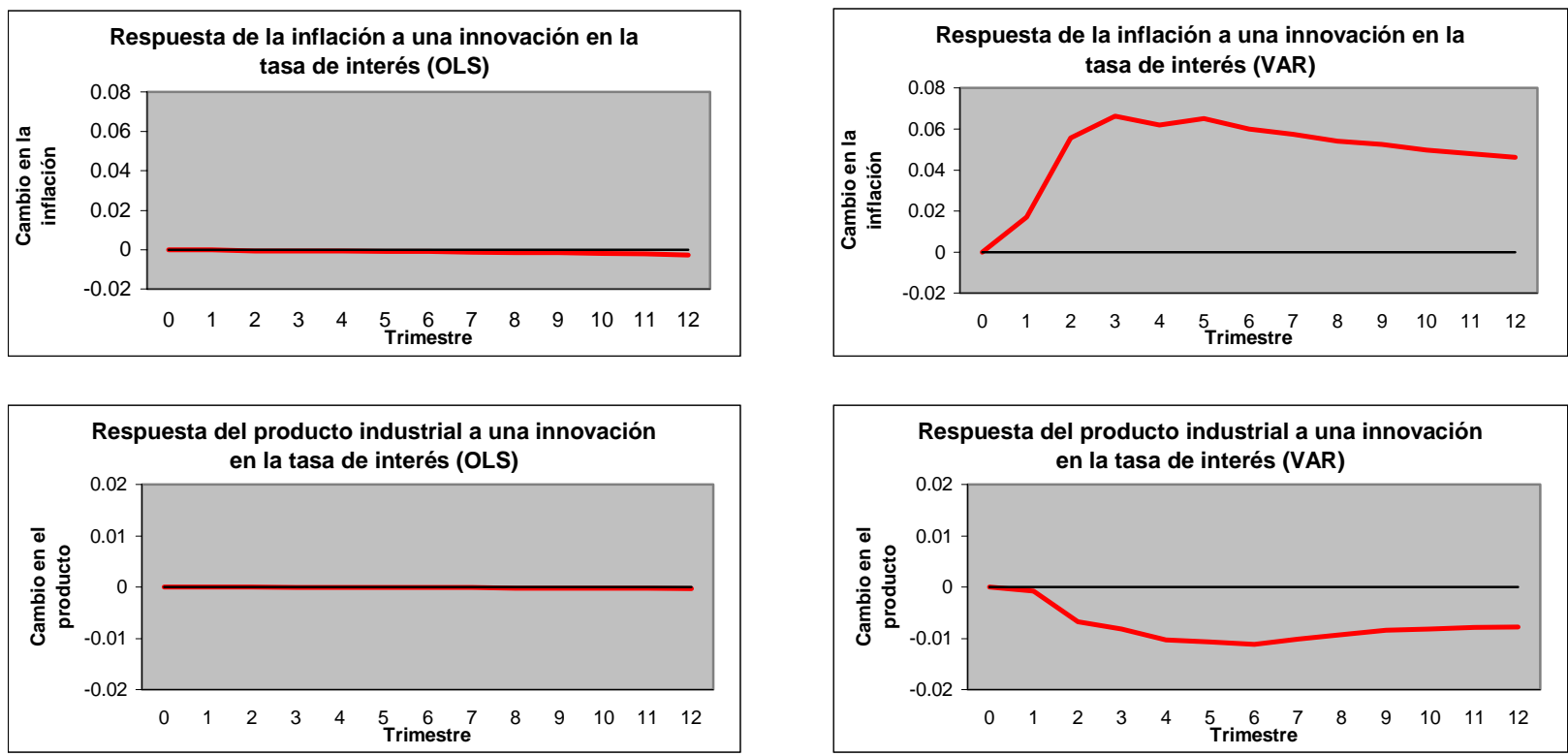

\section{Gráfica 3.2 Funciones de Impulso-Respuesta (Primer Subperiodo)}
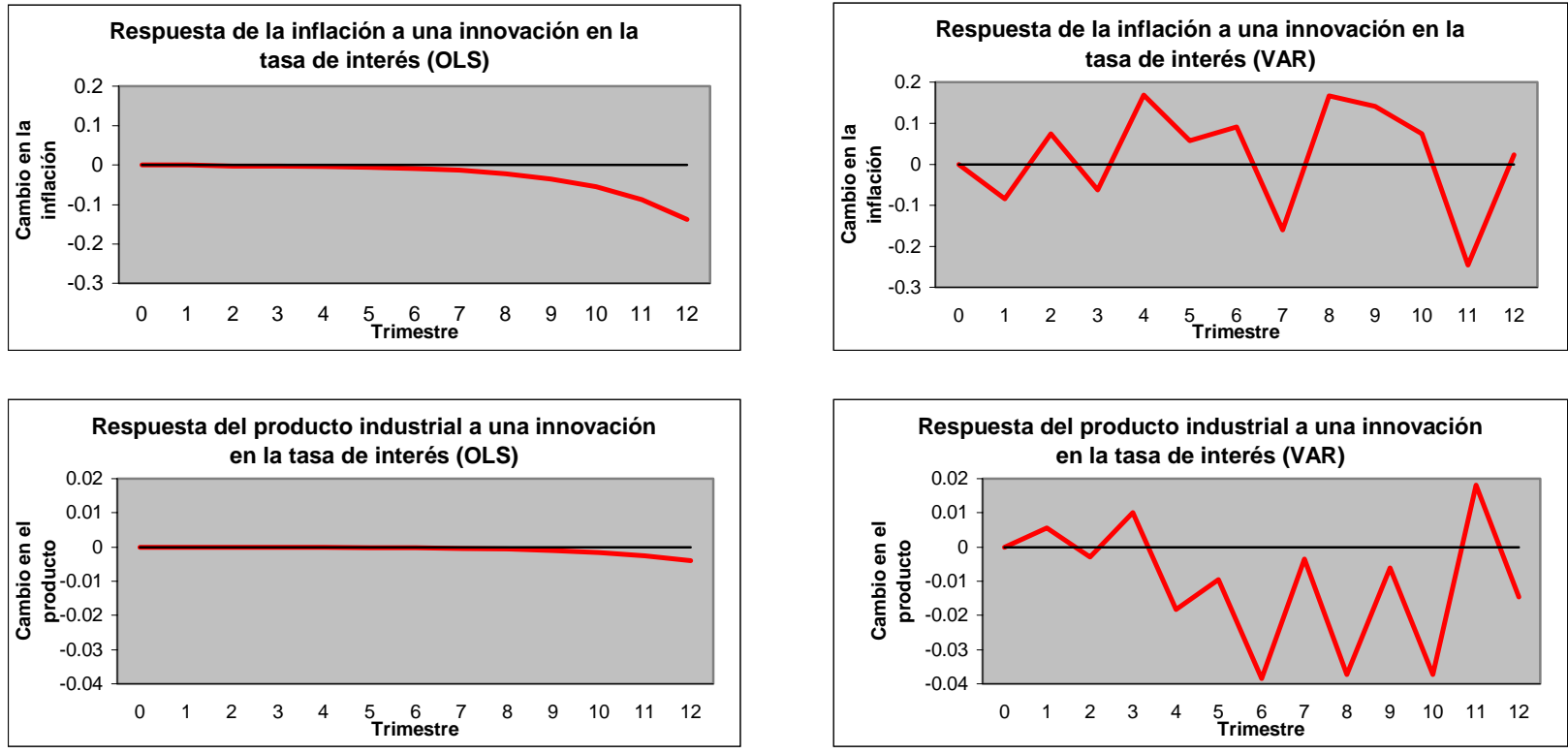

\footnotetext{
${ }^{10}$ En el caso del periodo completo y del primer subperiodo, las FIR no exhiben la forma de "U" mencionada. Sin embargo, tanto la inflación como el producto presentan una reducción inicial ante un incremento de la tasa de interés, lo cual es congruente con la teoría y contrasta con las FIR que se obtienen del modelo alternativo, como se detalla más adelante.
} 
Gráfica 3.3 Funciones de Impulso-Respuesta (Segundo Subperiodo)
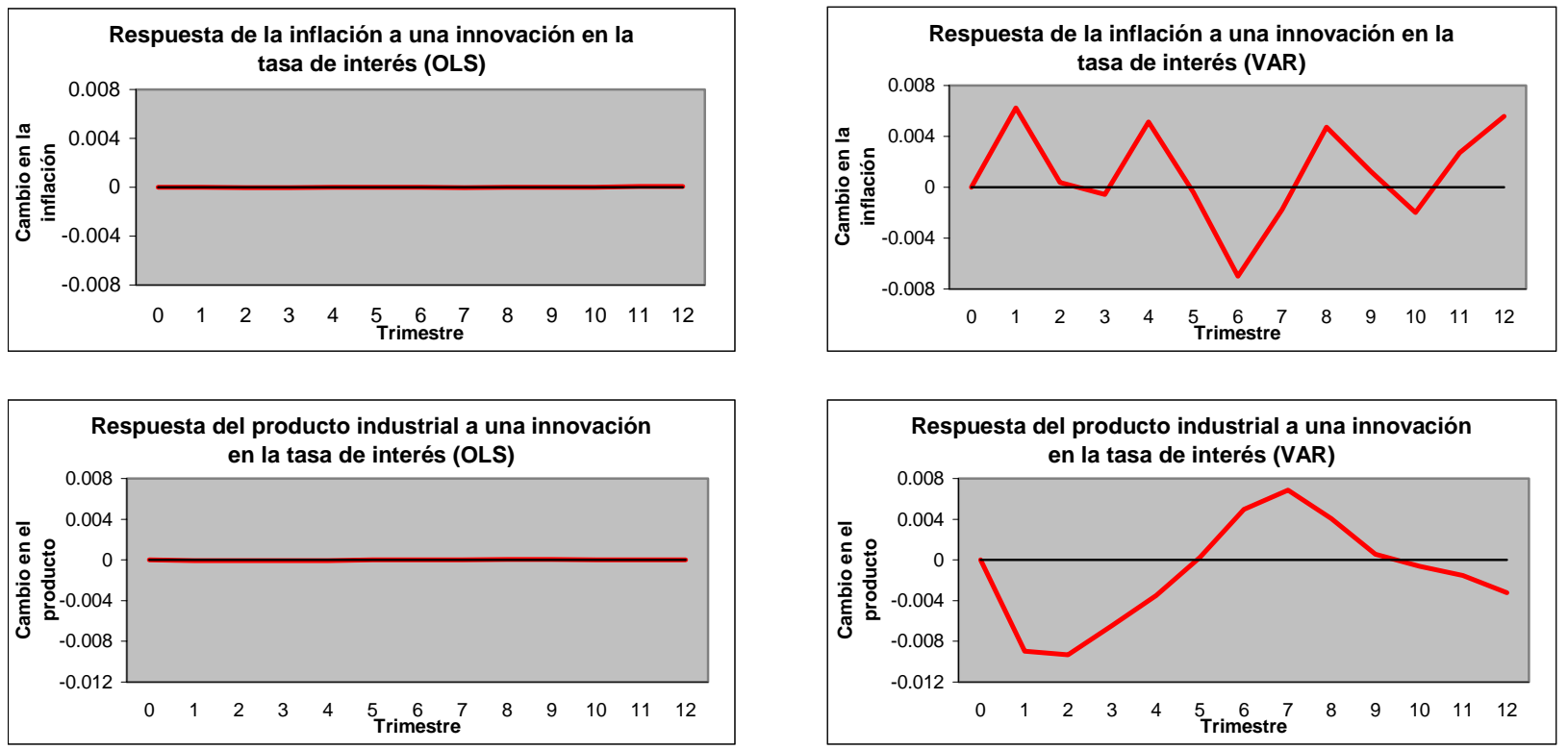

El desempeño del modelo estructural es contrastado con un modelo de vectores autorregresivos (VAR), el cual es frecuentemente utilizado en este tipo de análisis. Esta comparación resulta útil puesto que los modelos de VAR se pueden interpretar como resúmenes no teóricos de la dinámica de los datos. El modelo de VAR que se usa es del tipo irrestricto, incorporando tres variables endógenas (inflación, producción industrial y tasa de interés) con tres rezagos de cada una, además de tres variables exógenas dadas por $p x$, w y $c r$, para un total de 12 coeficientes a estimar para cada ecuación correspondiente a cada variable endógena. Por su parte, el modelo estructural se puede interpretar como un modelo de VAR restringido, en donde los respectivos coeficientes que no aparecen en el modelo estructural tienen valor de cero, e incluye una ecuación para la tasa de interés idéntica a la que contiene el modelo de VAR.

Una primera forma de comparar ambos modelos es a través de los criterios de información de Akaike (AIC) y Schwarz (SIC), los cuales son funciones de la suma de errores cuadrados y son empleados frecuentemente con el fin de seleccionar modelos. ${ }^{11}$ Ambos criterios favorecen al modelo estructural en el caso del primer subperiodo, mientras que para el segundo subperiodo y el periodo entero la evidencia es mixta: el criterio de SIC favorece al modelo estructural al tiempo que el criterio del AIC tiende a favorecer al modelo de VAR. Una diferencia importante entre el AIC y el SIC es que el SIC penaliza con mayor rigor el número de

\footnotetext{
${ }^{11}$ Los valores del AIC y el SIC se reportan en el Cuadro A.2 del apéndice.
} 
parámetros usados en contraste con el AIC. Dado el pequeño número de grados de libertad con los que se cuenta, se considera que el SIC es un mejor criterio para comparar estos modelos.

El segundo punto de comparación es con base en las FIR que resultan de ambos modelos. Como lo destacan Rudebusch y Svensson (1999), en vista de que la ecuación para la tasa de interés es idéntica en ambos modelos, cualquier discrepancia en las FIR será resultado de las restricciones impuestas a los coeficientes en el modelo estructural. Tal y como se mencionó, las FIR del modelo estructural son compatibles con lo que se espera de acuerdo a la teoría económica. Por su parte, las gráficas de las FIR del modelo de VAR para inflación no son congruentes con la teoría, pues se observa un aumento de la inflación al ocurrir un aumento en la tasa de interés. Este fenómeno, conocido en la literatura como price puzzle (ver Sims, 1992), ocurre con frecuencia al estimarse FIR en los modelos de VAR, y generalmente se puede solucionar al incluir como variable exógena un indicador líder de la inflación, como es el caso de un índice del precios de materias primas. Sin embargo, para el caso de México, el incorporar dicha variable no ayuda a solucionar el problema. ${ }^{12}$ De esta manera, a juzgar por las gráficas de las FIR, el modelo estructural utilizado en este estudio se perfila superior a un modelo de VAR.

\section{Derivación de la Frontera de Eficiencia}

En la presente sección se detalla la forma de construir la frontera de eficiencia, la cual es necesaria para evaluar el desempeño de la política monetaria. La frontera de eficiencia se deriva de la dinámica de la economía (dada por los coeficientes estimados del modelo estructural) y de una regla de política monetaria óptima, la cual es resultado de la minimización de una función de pérdida que representa los objetivos del Banco Central.

Suponemos que el objetivo de una regla óptima es minimizar la variabilidad de la inflación y del producto alrededor de una meta establecida por la autoridad monetaria para dichas variables. Por tanto, la frontera de eficiencia resulta útil en la medición del desempeño de la política monetaria al incorporar sus metas en conjunción con la estructura de la economía.

Si la regla de política monetaria no es óptima - por ejemplo, porque las autoridades monetarias no reaccionan de forma concordante con las perturbaciones en la economía- la política

\footnotetext{
${ }^{12}$ En la estimación del modelo de VAR se intentó revertir el price puzzle incluyendo, además del índice de precios de materias primas, el diferencial entre las tasas de interés de corto plazo doméstica y externa y la tasa de devaluación del peso. Ninguna de dichas variables, consideradas individualmente o en combinación con otras, permitió corregir el problema.
} 
monetaria puede ser más eficiente. Una caída en la variabilidad observada de la inflación y de la producción industrial puede ser, por ende, resultado de una política monetaria más eficiente; sin embargo, dicha caída puede deberse también a que la varianza de las perturbaciones a la economía se ha reducido.

Una manera de poder determinar la contribución relativa de cada una de estas dos posibilidades es a través del cálculo de la distancia más corta entre la frontera de eficiencia correspondiente y el punto de desempeño actual de la política monetaria para dos subperiodos diferentes. Para la estimación de dicha frontera se reescribió el modelo estructural en la siguiente representación de estado-espacio:

$$
\begin{gathered}
Y_{t+1}=B Y_{t}+c i_{t}+\delta X_{t}+v_{t+1} \\
\text { donde: } Y_{t}=\left[\begin{array}{c}
\pi_{t} \\
\pi_{t-1} \\
\pi_{t-2} \\
y_{t} \\
y_{t-1} \\
y_{t-2}
\end{array}\right] ; B=\left[\begin{array}{cccccc}
\alpha_{10} & \alpha_{11} & \alpha_{12} & \alpha_{13} & \alpha_{14} & 0 \\
1 & 0 & 0 & 0 & 0 & 0 \\
0 & 1 & 0 & 0 & 0 & 0 \\
0 \\
-\alpha_{23} & 0 & 0 & \alpha_{20} & \alpha_{21} & \alpha_{22} \\
0 & 0 & 0 & 1 & 0 & 0 \\
0 & 0 & 0 & 0 & 1 & 0
\end{array}\right] ; \\
c= \\
{\left[\begin{array}{c}
0 \\
\alpha_{23} \\
0 \\
0
\end{array}\right] ; v_{t+1}=\left[\begin{array}{c}
\varepsilon_{1 t} \\
0 \\
0 \\
\varepsilon_{2 t} \\
0 \\
0
\end{array}\right] ; \delta=\left[\begin{array}{cccc}
\alpha_{15} & \alpha_{16} & \alpha_{17} & 0 \\
0 & 0 & 0 & 0 \\
0 & 0 & 0 & 0 \\
0 & 0 & 0 & \alpha_{24} \\
0 & 0 & 0 & 0 \\
0 & 0 & 0 & 0
\end{array}\right] ; X_{t}=\left[\begin{array}{c}
p_{t} \\
w_{t} \\
c r_{t+1} \\
d e v_{t}
\end{array}\right]}
\end{gathered}
$$

Si el objetivo de las autoridades monetarias consiste en minimizar la variabilidad de la inflación y del producto respecto a sus niveles objetivo, éste se puede sintetizar en la minimización de la siguiente función de pérdida: ${ }^{13}$

$$
E[L]=E\left[\lambda\left(\pi_{t}-\pi^{*}\right)^{2}+(1-\lambda)\left(y_{t}-y^{*}\right)^{2}\right]
$$

donde $\pi^{*}$ es la meta de inflación de la autoridad monetaria, $y^{*}$ es el producto potencial de la economía, y $\lambda$ mide la importancia relativa que la autoridad monetaria da a cada una de estas variabilidades. ${ }^{14} \mathrm{Si}$ se supone que los valores meta de la autoridad monetaria son

\footnotetext{
${ }^{13}$ Esta función de pérdida no incluye el tipo de cambio o alguna tasa de interés, pues se supone que la preocupación principal de la autoridad monetaria es el desempeño macroeconómico interno, el cual se evalúa a través de la estabilidad de precios y del producto.

${ }^{14} \mathrm{El}$ parámetro $\lambda$ se puede interpretar como una medida de aversión a la variabilidad de la inflación por parte de la autoridad monetaria (ver Cecchetti y Ehrmann, 1999).
} 
iguales a los promedios históricos de cada variable, la anterior ecuación se puede reescribir de la siguiente forma:

$$
E[L]=\lambda \operatorname{var}\left(\pi_{t}\right)+(1-\lambda) \operatorname{var}\left(y_{t}\right)
$$

y ésta, a su vez, en notación matricial como:

$$
E\left[Y_{t}^{\prime} \Lambda Y_{t}\right]
$$

donde:

$$
\Lambda=\left[\begin{array}{cccccc}
\lambda & 0 & 0 & 0 & 0 & 0 \\
0 & 0 & 0 & 0 & 0 & 0 \\
0 & 0 & 0 & 0 & 0 & 0 \\
0 & 0 & 0 & 1-\lambda & 0 & 0 \\
0 & 0 & 0 & 0 & 0 & 0 \\
0 & 0 & 0 & 0 & 0 & 0
\end{array}\right]
$$

El problema de control óptimo se completa a través de la siguiente ecuación para la variable de control (tasa de interés):

$$
i_{t}=\Gamma Y_{t}+\Psi_{t}
$$

donde $\Gamma$ es el vector de coeficientes de reacción de la autoridad monetaria a cambios en la inflación y el producto y $\Psi_{t}$ es una expresión que depende de $B, c, X, \pi^{*}$ y $y^{*} .^{15}$ La ecuación (10) es la forma supuesta para la regla de política monetaria, la cual se conoce como de tipo irrestricta (ver Rudebusch y Svensson, 1999).

De manera formal, el problema de control óptimo es el de encontrar un camino óptimo para la variable de control (tasa de interés) de la ecuación (10) que minimice la ecuación (8), sujeta a la dinámica de la economía (ecuación (5)). El parámetro $\Gamma$ se obtiene como solución al siguiente sistema de ecuaciones: ${ }^{16}$

$$
\Gamma=-\left(c^{\prime} H c\right)^{-1} c^{\prime} H B
$$

donde:

$$
H=\Lambda+(B+c \Gamma)^{\prime} H(B+c \Gamma)
$$

Con el fin de estimar $\Gamma$, se calculó su valor de manera iterativa, empleando los coeficientes estimados de $B$ y $c$ en la sección anterior y usando la matriz $\Lambda$ como valor inicial para la matriz $H$.

\footnotetext{
${ }^{15}$ Ver Chow (1975), pp. 158-159.

${ }^{16}$ Para una exposición técnica de cómo resolver este problema de control óptimo el lector es referido a Taylor (1979), el cual usa técnicas de Chow (1975).
} 
El último paso consiste en calcular las varianzas óptimas de la inflación y del producto para distintos valores de $\lambda$, las cuales se derivan del primer y cuarto elementos diagonales de la matriz de varianza-covarianza de $Y_{t}$ en su estado estacionario. Una vez obtenidas dichas varianzas, es posible trazar la frontera de eficiencia graficando los pares de varianzas óptimas en función de $\lambda$.

\section{Medidas de eficiencia de la política monetaria}

La medidas propuestas para determinar si la política monetaria se ha vuelto más eficiente requieren de la estimación del modelo estructural y de la frontera de eficiencia para ambos subperiodos, además del cálculo de las variabilidades observadas de la inflación y del producto.

La primera medida se determina calculando la distancia euclidiana entre el par de variabilidades observadas de inflación y producto y la frontera, la cual se denota como $\mu_{i}(i=1,2$; subperiodos; ver Gráfica 4). En el caso de que la diferencia entre estas dos distancias, definida como $\mu=\mu_{1}-\mu_{2}$, resulte positiva, ello se interpretaría como un incremento en la eficiencia de la política monetaria del subperiodo 1 al subperiodo 2; mientras que en el caso de que $\mu$ fuese negativa, existiría una pérdida de eficiencia entre subperiodos.

La segunda medida busca establecer la contribución relativa de una política monetaria más eficiente al cambio observado en las variabilidades de la inflación y del producto entre un subperiodo y otro. Para ello, se define inicialmente (ver Gráfica 4) la métrica $m_{i}(i=1,2$; subperiodos) como la distancia euclidiana entre el par de variabilidades observadas para cada subperiodo y el origen. Análogamente, se define la métrica $n_{i}(i=1,2$; subperiodos) como la distancia entre el par de variabilidades óptimas a partir de las cuales se minimiza la distancia entre la curva y el punto de desempeño actual, y el origen. 


\section{Gráfica 4: Distancias}

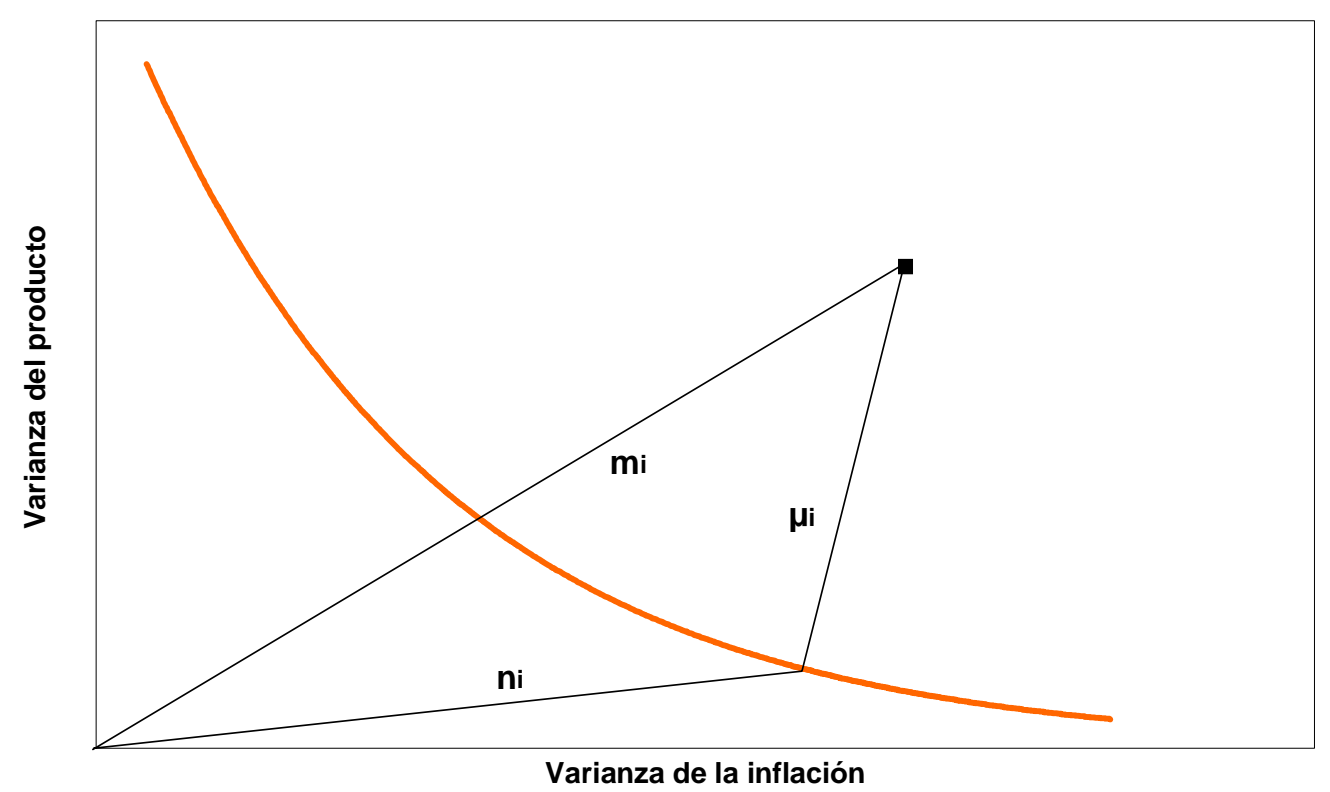

La diferencia $m=m_{1}-m_{2}$ brinda una medida de la reducción (o incremento, en caso de ser negativa) de la variabilidad observada del producto y de la inflación de un subperiodo a otro, mientras que la diferencia $n=n_{1}-n_{2}$ indica cual ha sido el desplazamiento hacia adentro (o hacia afuera, en caso de que $n$ sea negativa) de la frontera de eficiencia. Por lo tanto, la contribución relativa de la política monetaria a la reducción de la variabilidad observada en los datos estará dada por el siguiente cociente:

$$
q=\frac{\left(m_{1}-m_{2}\right)-\left(n_{1}-n_{2}\right)}{\left(m_{1}-m_{2}\right)}=1-\frac{\left(n_{1}-n_{2}\right)}{\left(m_{1}-m_{2}\right)}
$$

En la siguiente sección se procede a estimar tanto $\mu$ como $q$ con los datos de la economía mexicana con el fin de determinar si existe algun aumento en la eficiencia de la política monetaria en México y, de ser este el caso, cual es su importancia relativa en explicar la caída observada en la variabilidad de la inflación y del producto.

\section{Resultados}

Analizando el desempeño actual de la economía mexicana, tal y como se mencionó en la introducción, la varianza de la inflación trimestral anualizada pasó de 7.02\% en el primer subperiodo a $1.76 \%$ en el segundo, mientras que la varianza del logaritmo natural de la producción industrial se redujo de $0.40 \%$ a $0.19 \%$. 
En la Gráfica 5 se presenta la frontera de eficiencia junto al punto actual de desempeño de la política monetaria para los dos subperiodos en estudio. Comparando ambas curvas, es evidente que la frontera se ha desplazado hacia adentro en el segundo subperiodo, lo cual implica una reducción en la varianza de las perturbaciones de oferta del primero al segundo subperiodo. Adicionalmente, se observa que la frontera cambia de forma, lo cual se debe a las diferentes estructuras de la economía entre subperiodos. Siguiendo a Cecchetti y Ehrmann (1999), la frontera de eficiencia para el primer subperiodo está relacionada con una curva de oferta agregada plana, mientras que la correspondiente al segundo subperiodo con una curva de oferta agregada con mayor pendiente. En efecto, los valores correspondientes a la pendiente de la curva de oferta agregada son 0.06 y 0.43 para el primero y segundo subperiodos, respectivamente. ${ }^{17,18}$

\section{Gráfica 5: Frontera de Eficiencia y Punto de Desempeño (Comparación entre subperiodos)}

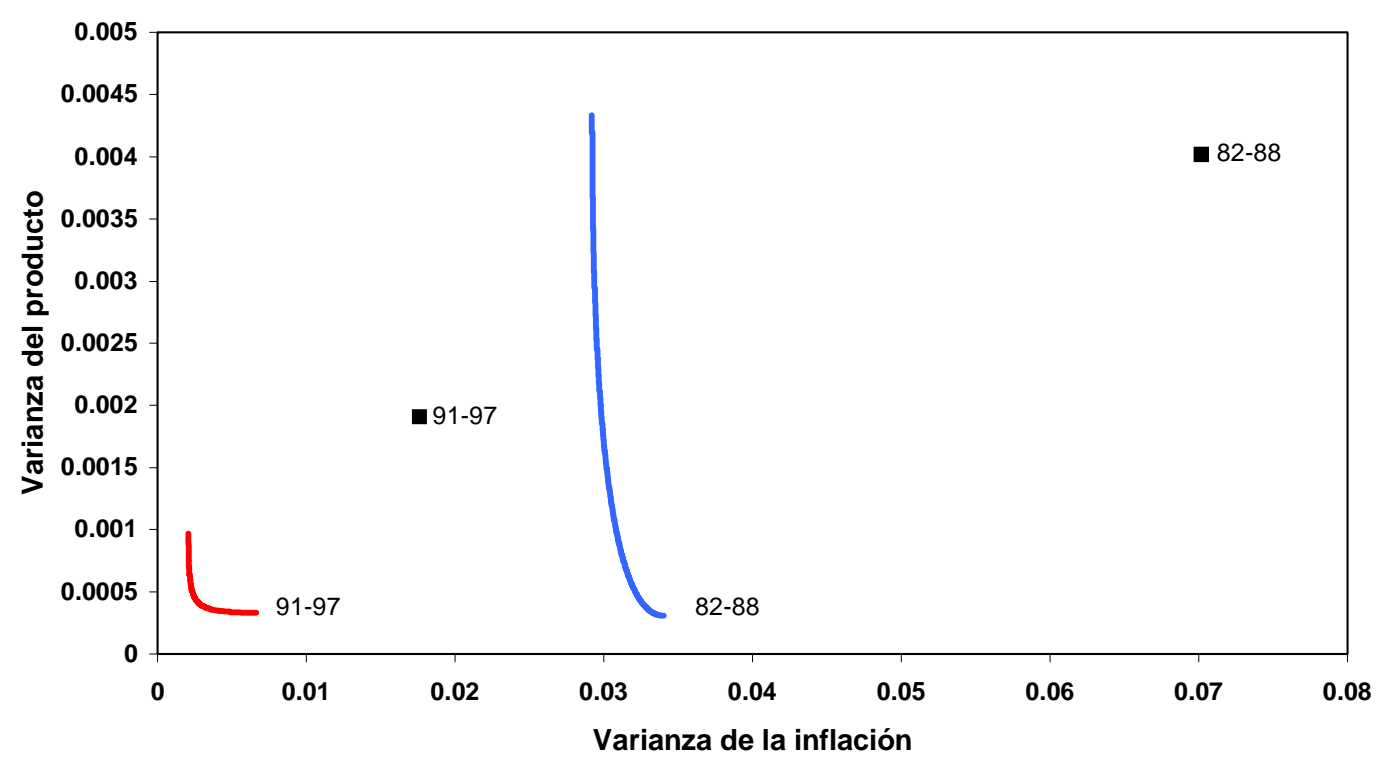

En vista de que la variabilidad observada en los datos se ha reducido y al mismo tiempo la frontera se ha movido hacia adentro, se utilizaron las medidas introducidas en la sección

\footnotetext{
${ }^{17}$ Siguiendo a Cecchetti y Ehrmann (1999), se calcula la pendiente de la curva de oferta agregada como la razón entre el promedio de 12 trimestres del impacto de la tasa nominal de interés sobre el producto y el impacto de dicha razón sobre la tasa de inflación.

${ }^{18} \mathrm{El}$ Cuadro A.3 del apéndice presenta los valores óptimos de las varianzas de la inflación y de la producción industrial para ciertos valores de $\lambda$; mientras que el Cuadro A.4 reporta los valores de $\Gamma$ para los mismos valores de $\lambda$.
} 
anterior para establecer si la reducción en la variabilidad observada se debe, al menos en parte, a una política monetaria más eficiente. A partir de la derivación de la frontera de eficiencia y la varianza observada en los datos es posible determinar que $\mu_{1}=0.0364$ para el primer subperiodo, mientras que para el segundo $\mu_{2}=0.0207$, lo cual implica que $\mu=0.0157$. El signo positivo de $\mu$, como se señaló en la sección anterior, indica que la política monetaria en México ha sido más eficiente en la década de los noventa respecto a su desempeño durante los ochenta.

Por medio de la segunda medida propuesta es posible estimar la proporción de la reducción en la varianza observada de la inflación y del producto que se debe a un mejor manejo de la política monetaria. Empleando las medidas definidas en la sección anterior, se observa que $\left(m_{1} ; m_{2} ; n_{1} ; n_{2}\right)=(0.0703 ; 0.0177 ; 0.0339 ; 0.0063)$. A partir de estos datos y utilizando la fórmula de la ecuación (12), se obtiene el valor $q=0.4740$, con el cual se concluye que la contribución relativa de una mejor política monetaria a la reducción observada en la varianza del producto y de la inflación es igual a $47 \%$.

Al calcular las varianzas observadas de la inflación y el producto se supone que el objetivo del Banco de México consiste en reducir la volatilidad de dichas variables alrededor de su promedio histórico. En el caso de la inflación, para el primer subperiodo la tasa trimestral promedio anualizada de la inflación es de $62.30 \%$, mientras que para el segundo subperiodo es de $17.93 \%$. Dichas cifras distan significativamente de una meta razonable para la inflación. Si alternativamente consideramos que el objetivo de mediano plazo del Banco de México desde 1999 es el de alcanzar una tasa de inflación igual a la de sus principales socios comerciales para 2003, y se supone que dicha meta de inflación es la correspondiente a los Estados Unidos (principal socio comercial de México), los valores objetivo de la inflación serían 3.58\% para el primer subperiodo y $2.70 \%$ para el segundo. ${ }^{19}$

Utilizando estos últimos datos las variabilidades de la inflación son de $42.78 \%$ para el primer subperiodo y de $4.17 \%$ para el segundo. El valor de $\mu_{1}$ aumenta a 0.3939 mientras que el de $\mu_{2}$ pasa a 0.0544, con lo que $\mu$ alcanza un valor igual a 0.3395; es decir, la eficiencia de la política monetaria se incrementa en valor absoluto, reforzando la evidencia de un mejor manejo de la política monetaria en México durante la década de los noventa. Adicionalmente, para la segunda medida los valores de $\left(m_{1} ; m_{2} ; n_{1} ; n_{2}\right)$ cambian a $(0.4279 ; 0.0417 ; 0.0339 ; 0.0063)$, resultando en una contribución relativa de la política monetaria que alcanza $93 \%$.

\footnotetext{
${ }^{19}$ Este objetivo de mediano plazo se encuentra citado en el documento oficial del Banco de México "Política Monetaria: Programa para 2000".
} 


\section{Conclusiones}

En comparación con la década de los ochenta, México ha experimentado una mayor estabilidad macroeconómica - en lo que respecta a las variabilidades de la inflación y de la producción industrial- durante la década de los noventa. Esta mayor estabilidad ha sido el resultado combinado de una reducción en la varianza de las perturbaciones de oferta agregada y de una política monetaria más eficiente. Con el fin de determinar la contribución relativa de cada uno de estos factores, se calculó la frontera de eficiencia para dos subperiodos y se desarrollaron medidas de ganancia de eficiencia netas del cambio en la varianza de las perturbaciones de oferta.

Utilizando un modelo estructural de oferta y demanda agregadas para la economía mexicana -cuyo objetivo es estimar la frontera de eficiencia- se establece que, en efecto, dicha frontera se ha desplazado hacia adentro, indicando que la varianza de las perturbaciones de oferta se ha reducido en la década de los noventa. Empleando las medidas propuestas es posible determinar que al mismo tiempo se ha presentado un incremento en la eficiencia de la política monetaria, cuya contribución relativa es de $47 \%$ si se considera que el objetivo de la política monetaria es el de minimizar la varianza de la inflación y del producto (alrededor de sus respectivos promedios históricos). Este resultado es aún más claro si se supone que el objetivo de la política monetaria consiste en mantener la tasa de inflación lo más cercana posible a la de su principal socio comercial, los Estados Unidos. Utilizando este supuesto alternativo, la contribución relativa de una política monetaria más eficiente en la consecución de una mayor estabilidad macroeconómica alcanza $93 \%$.

En síntesis, los resultados del presente documento de investigación presentan evidencia de una significativa contribución del aumento en la eficiencia en la política monetaria en México durante los últimos años para alcanzar una mayor estabilidad macroeconómica, tomando en cuenta la reducción de la varianza de las perturbaciones de oferta. 


\section{Bibliografía}

Andrews, D. W. K. (1993). "Tests for Parameter Instability and Structural Change with Unknown Change Point", Econometrica 61 (4), pp. 821-856.

Cecchetti, S. G. y M. Ehrmann (1999). "Does Inflation Targeting Increase Output Volatility? An International Comparison of Policymakers' Preferences and Outcomes", NBER Working Paper 7426.

Chow, G. C. (1975). Analysis and Control of Dynamic Economic Systems, John Wiley and Sons, New York.

Garcés Díaz, D. G. (1999). "Determinación del Nivel de Precios y la Dinámica Inflacionaria en México", Documento de Investigación No. 9907, Banco de México.

Rudebusch, G. D. y L. E. O. Svensson (1999) "Policy Rules for Inflation Targeting" in J. B. Taylor ed. Monetary Policy Rules, University of Chicago Press, Chicago, pp. 203-246.

Sims, C. (1992). "Interpreting the Macroeconomic Time Series Facts: The Effects of Monetary Policy“, European Economic Review, 36 (5), pp. 975-1000.

Taylor, J. B. (1979). "Estimation and Control of a Macroeconomic Model with Rational Expectations", Econometrica, 47, pp. 1267-1286. 


\section{Apéndice}

\section{Cuadro A.1: Coeficientes Estimados del Modelo Estructural}

\begin{tabular}{|c|c|c|c|c|c|c|c|c|}
\hline \multicolumn{3}{|c|}{$\begin{array}{c}\text { Primer Subperiodo } \\
(1982-1988)\end{array}$} & \multicolumn{3}{|c|}{$\begin{array}{c}\text { Segundo Subperiodo } \\
(1991-1997)\end{array}$} & \multicolumn{3}{|c|}{$\begin{array}{c}\text { Periodo Completo } \\
(1982-1997)\end{array}$} \\
\hline \multirow{2}{*}{$\begin{array}{l}\text { Variables } \\
\text { indep. }\end{array}$} & \multicolumn{2}{|c|}{ Variables dep. } & \multirow{2}{*}{$\begin{array}{l}\text { Variables } \\
\text { indep. }\end{array}$} & \multicolumn{2}{|c|}{ Variables dep. } & \multirow{2}{*}{$\begin{array}{l}\text { Variables } \\
\text { indep. }\end{array}$} & \multicolumn{2}{|c|}{ Variables dep. } \\
\hline & $\pi(t+1)$ & $y(t+1)$ & & \multirow{2}{*}{\multicolumn{2}{|c|}{$\frac{\pi(t+1)}{0.4997}$}} & & \multicolumn{2}{|l|}{$\pi(t+1)$} \\
\hline$\pi(t)$ & \multicolumn{2}{|l|}{0.5862} & $\pi(t)$ & & & $\pi(t)$ & \\
\hline $\begin{array}{c}\text { Desv. Est. } \\
\text { Valor } p\end{array}$ & \multicolumn{2}{|l|}{0.1811} & $\begin{array}{c}\text { Desv. Est. } \\
\text { Valor } p\end{array}$ & \multicolumn{2}{|l|}{$\begin{array}{l}0.1009 \\
0.0000\end{array}$} & $\begin{array}{c}\text { Desv. Est. } \\
\text { Valor } p\end{array}$ & \multicolumn{2}{|l|}{$\begin{array}{l}0.0992 \\
0.0000\end{array}$} \\
\hline$\pi(t-1)$ & \multicolumn{2}{|l|}{$\frac{0.0012}{-0.0891}$} & $\pi(t-1)$ & \multicolumn{2}{|l|}{0.0104} & $\pi(t-1)$ & \multicolumn{2}{|l|}{-0.0934} \\
\hline $\begin{array}{l}\text { Desv. Est. } \\
\text { Valor } p\end{array}$ & \multicolumn{2}{|l|}{0.2027} & $\begin{array}{l}\text { Desv. Est. } \\
\text { Valor } p\end{array}$ & \multicolumn{2}{|l|}{$\begin{array}{l}0.1420 \\
0.9419\end{array}$} & $\begin{array}{l}\text { Desv. Est. } \\
\text { Valor } p\end{array}$ & \multicolumn{2}{|l|}{$\begin{array}{l}0.1170 \\
0.9767\end{array}$} \\
\hline$\pi(\mathrm{t}-2)$ & \multicolumn{2}{|l|}{$\frac{0.6605}{0.1484}$} & $\pi(\mathrm{t}-2)$ & \multicolumn{2}{|l|}{0.4184} & $\pi(\mathrm{t}-2)$ & & \\
\hline $\begin{array}{l}\text { Desv. Est. } \\
\text { Valor } p\end{array}$ & \multicolumn{2}{|l|}{$\begin{array}{l}0.1758 \\
0.3986\end{array}$} & $\begin{array}{l}\text { Desv. Est. } \\
\text { Valor } p\end{array}$ & \multicolumn{2}{|l|}{$\begin{array}{l}0.1292 \\
0.0012\end{array}$} & $\begin{array}{l}\text { Desv. Est. } \\
\text { Valor } p\end{array}$ & \multicolumn{2}{|l|}{$\begin{array}{l}0.0921 \\
0.2080\end{array}$} \\
\hline$y(t)$ & 1.5550 & 1.1344 & $y(t)$ & 0.8758 & 1.0798 & $y(t)$ & 0.9700 & 1.0562 \\
\hline $\begin{array}{l}\text { Desv. Est. } \\
\text { Valor } p\end{array}$ & $\begin{array}{l}2.0990 \\
0.4589\end{array}$ & $\begin{array}{l}0.2617 \\
0.0000\end{array}$ & $\begin{array}{l}\text { Desv. Est. } \\
\text { Valor } p\end{array}$ & $\begin{array}{l}0.6493 \\
0.1774\end{array}$ & $\begin{array}{l}0.2210 \\
0.0000\end{array}$ & $\begin{array}{l}\text { Desv. Est. } \\
\text { Valor } p\end{array}$ & $\begin{array}{l}0.9061 \\
0.2844\end{array}$ & $\begin{array}{l}0.1243 \\
0.0000\end{array}$ \\
\hline$y(t-1)$ & \multirow{3}{*}{$\begin{array}{r}\mathbf{- 1 . 2 2 9 0} \\
1.9550 \\
0.5298 \\
\end{array}$} & \multirow{3}{*}{$\begin{array}{r}-0.0335 \\
0.3499 \\
0.9238 \\
-0.2921\end{array}$} & $y(t-1)$ & 0.2187 & -0.1180 & $y(t-1)$ & -0.5041 & -0.0263 \\
\hline $\begin{array}{l}\text { Desv. Est. } \\
\text { Valor } p\end{array}$ & & & $\begin{array}{l}\text { Desv. Est. } \\
\text { Valor } p\end{array}$ & $\begin{array}{l}0.5899 \\
0.7108\end{array}$ & $\begin{array}{l}0.3244 \\
0.7160\end{array}$ & $\begin{array}{l}\text { Desv. Est. } \\
\text { Valor } p\end{array}$ & $\begin{array}{l}0.8330 \\
0.5451\end{array}$ & $\begin{array}{l}0.1893 \\
0.8894\end{array}$ \\
\hline$y(t-2)$ & & & $y(t-2)$ & & -0.3012 & $y(t-2)$ & & -0.2769 \\
\hline $\begin{array}{c}\text { Desv. Est. } \\
\text { Valor } p\end{array}$ & & $\begin{array}{l}0.2102 \\
0.1647\end{array}$ & $\begin{array}{l}\text { Desv. Est. } \\
\text { Valor } p\end{array}$ & & $\begin{array}{l}0.1984 \\
0.1289\end{array}$ & $\begin{array}{l}\text { Desv. Est. } \\
\text { Valor } p\end{array}$ & & $\begin{array}{l}0.1168 \\
0.0177\end{array}$ \\
\hline $\mathrm{px}(\mathrm{t})$ & 0.1181 & & $p x(t)$ & 0.0549 & & $p x(t)$ & 0.0764 & \\
\hline $\begin{array}{l}\text { Desv. Est. } \\
\text { Valor } p\end{array}$ & $\begin{array}{l}0.0558 \\
0.0344\end{array}$ & & $\begin{array}{l}\text { Desv. Est. } \\
\text { Valor } p\end{array}$ & $\begin{array}{l}0.0091 \\
0.0000\end{array}$ & & $\begin{array}{l}\text { Desv. Est. } \\
\text { Valor } p\end{array}$ & $\begin{array}{l}0.0152 \\
0.0000\end{array}$ & \\
\hline $\mathbf{w}(\mathrm{t})$ & 0.0641 & & $\mathrm{w}(\mathrm{t})$ & 0.0201 & & $w(t)$ & 0.0517 & \\
\hline $\begin{array}{l}\text { Desv. Est. } \\
\text { Valor }\end{array}$ & 0.0168 & & Desv. Est. & $\begin{array}{l}0.0069 \\
0.0036\end{array}$ & & $\begin{array}{l}\text { Desv. Est. } \\
\text { Valor }\end{array}$ & 0.0082 & \\
\hline$i(t)-\pi(t)$ & & -0.0052 & $i(t)-\pi(t)$ & & -0.0762 & $i(t)-\pi(t)$ & & -0.0190 \\
\hline $\begin{array}{l}\text { Desv. Est. } \\
\text { Valor } p\end{array}$ & & $\begin{array}{l}0.0290 \\
0.8575\end{array}$ & $\begin{array}{l}\text { Desv. Est. } \\
\text { Valor } p\end{array}$ & & $\begin{array}{l}0.0768 \\
0.3209\end{array}$ & $\begin{array}{l}\text { Desv. Est. } \\
\text { Valor } p\end{array}$ & & $\begin{array}{l}0.0206 \\
0.3549\end{array}$ \\
\hline $\operatorname{dev}(t)$ & & -0.0056 & $\operatorname{dev}(t)$ & & -0.0058 & $\operatorname{dev}(t)$ & & -0.0064 \\
\hline $\begin{array}{l}\text { Desv. Est. } \\
\text { Valor } p\end{array}$ & & $\begin{array}{l}0.0060 \\
0.3518\end{array}$ & $\begin{array}{l}\text { Desv. Est. } \\
\text { Valor } p\end{array}$ & & $\begin{array}{l}0.0034 \\
0.0895\end{array}$ & $\begin{array}{l}\text { Desv. Est. } \\
\text { Valor } p\end{array}$ & & $\begin{array}{l}0.0023 \\
0.0053\end{array}$ \\
\hline $\operatorname{cr}(\mathrm{t}+1)$ & 0.0566 & & $\operatorname{cr}(\mathrm{t}+1)$ & 0.1344 & & $\operatorname{cr}(\mathrm{t}+1)$ & 0.0222 & \\
\hline $\begin{array}{l}\text { Desv. Est. } \\
\text { Valor } p\end{array}$ & $\begin{array}{l}0.1250 \\
0.6503\end{array}$ & & $\begin{array}{l}\text { Desv. Est. } \\
\text { Valor } p\end{array}$ & $\begin{array}{l}0.0488 \\
0.0058\end{array}$ & & $\begin{array}{l}\text { Desv. Est. } \\
\text { Valor } p\end{array}$ & $\begin{array}{l}0.0543 \\
0.6830\end{array}$ & \\
\hline R2 ajust. & 0.5809 & 0.7961 & R2 ajust. & 0.8806 & 0.8320 & R2 ajust. & 0.8371 & 0.8424 \\
\hline
\end{tabular}




\section{Cuadro A.2: Comparación de Modelos Usando AIC y SIC}

\begin{tabular}{|c|c|c|c|c|}
\hline Primer Subperiodo & \multicolumn{2}{|c|}{ AIC } & \multicolumn{2}{c|}{ SIC } \\
\hline & $\begin{array}{c}\text { Ecuación de } \\
\text { Inflación }\end{array}$ & $\begin{array}{c}\text { Ecuación de Producción } \\
\text { Industrial }\end{array}$ & $\begin{array}{c}\text { Ecuación de } \\
\text { Inflación }\end{array}$ & $\begin{array}{c}\text { Ecuación de Producción } \\
\text { Industrial }\end{array}$ \\
\hline Modelo Estructural & -3.18 & -7.73 & -2.79 & -7.49 \\
\hline Modelo de VAR & -3.01 & -7.76 & -2.42 & -7.17 \\
\hline
\end{tabular}

\begin{tabular}{|c|c|c|c|c|}
\hline Segundo Subperiodo & \multicolumn{2}{|c|}{ AIC } & \multicolumn{2}{c|}{ SIC } \\
\hline & $\begin{array}{c}\text { Ecuación de } \\
\text { Inflación }\end{array}$ & $\begin{array}{c}\text { Ecuación de Producción } \\
\text { Industrial }\end{array}$ & $\begin{array}{c}\text { Ecuación de } \\
\text { Inflación }\end{array}$ & $\begin{array}{c}\text { Ecuación de Producción } \\
\text { Industrial }\end{array}$ \\
\hline Modelo Estructural & -5.83 & -7.75 & -5.44 & -7.50 \\
\hline Modelo de VAR & -6.13 & -8.07 & -5.54 & -7.49 \\
\hline
\end{tabular}

\begin{tabular}{|c|c|c|c|c|}
\hline Periodo Completo & \multicolumn{2}{|c|}{ AIC } & \multicolumn{2}{c|}{ SIC } \\
\hline & $\begin{array}{c}\text { Ecuación de } \\
\text { Inflación }\end{array}$ & $\begin{array}{c}\text { Ecuación de Producción } \\
\text { Industrial }\end{array}$ & $\begin{array}{c}\text { Ecuación de } \\
\text { Inflación }\end{array}$ & $\begin{array}{c}\text { Ecuación de Producción } \\
\text { Industrial }\end{array}$ \\
\hline Modelo Estructural & -4.14 & -8.01 & -3.86 & -7.83 \\
\hline Modelo de VAR & -4.16 & -8.11 & -3.74 & -7.69 \\
\hline
\end{tabular}

\section{Cuadro A.3: Variabilidades Óptimas de la Inflación y del Producto}

\begin{tabular}{|c|c|c|c|c|}
\hline & \multicolumn{2}{|c|}{ Varianza de la Inflación (\%) } & \multicolumn{2}{c|}{ Varianza del Producto (\%) } \\
\hline$\lambda$ & $\begin{array}{c}\text { Primer } \\
\text { Subperiodo }\end{array}$ & $\begin{array}{c}\text { Segundo } \\
\text { Subperiodo }\end{array}$ & $\begin{array}{c}\text { Primer } \\
\text { Subperiodo }\end{array}$ & $\begin{array}{c}\text { Segundo } \\
\text { Subperiodo }\end{array}$ \\
\hline 0.1 & 3.286 & 0.267 & 0.035 & 0.036 \\
\hline 0.2 & 3.195 & 0.238 & 0.051 & 0.041 \\
\hline 0.3 & 3.122 & 0.225 & 0.076 & 0.045 \\
\hline 0.4 & 3.062 & 0.218 & 0.108 & 0.049 \\
\hline 0.5 & 3.013 & 0.213 & 0.147 & 0.053 \\
\hline 0.6 & 2.976 & 0.209 & 0.193 & 0.058 \\
\hline 0.7 & 2.948 & 0.206 & 0.244 & 0.063 \\
\hline 0.8 & 2.929 & 0.204 & 0.301 & 0.068 \\
\hline 0.9 & 2.918 & 0.203 & 0.365 & 0.076 \\
\hline
\end{tabular}


Cuadro A.4: Coeficientes de Reacción de la Regla de Política Óptima $(\Gamma)$

\begin{tabular}{|c|c|c|c|c|c|c|c|c|c|c|}
\hline \multirow[b]{2}{*}{$\lambda$} & \multicolumn{5}{|c|}{ Primer Subperiodo } & \multicolumn{5}{|c|}{ Segundo Subperiodo } \\
\hline & $\pi(t)$ & $\pi(t-1)$ & $\pi(t-2)$ & $y(t)$ & $y(t-1)$ & $\pi(t)$ & $\pi(t-1)$ & $\pi(t-2)$ & $y(t)$ & $y(t-1)$ \\
\hline 0.1 & 6.70 & 2.14 & 1.93 & 211.0 & -22.42 & 1.29 & 0.50 & 0.34 & 8.27 & -0.62 \\
\hline 0.2 & 10.79 & 3.87 & 3.49 & 205.2 & -35.37 & 1.61 & 0.80 & 0.49 & 8.75 & -0.54 \\
\hline 0.3 & 14.46 & 5.34 & 4.81 & 200.3 & -46.27 & 1.83 & 1.07 & 0.62 & 9.15 & -0.47 \\
\hline 0.4 & 17.81 & 6.60 & 5.95 & 196.1 & -55.70 & 2.00 & 1.33 & 0.73 & 9.52 & -0.42 \\
\hline 0.5 & 20.88 & 7.71 & 6.95 & 192.4 & -64.03 & 2.15 & 1.59 & 0.84 & 9.88 & -0.36 \\
\hline 0.6 & 23.75 & 8.71 & 7.86 & 189.2 & -71.50 & 2.27 & 1.86 & 0.95 & 10.25 & -0.30 \\
\hline 0.7 & 26.42 & 9.61 & 8.67 & 186.2 & -78.27 & 2.37 & 2.15 & 1.07 & 10.65 & -0.24 \\
\hline 0.8 & 28.93 & 10.44 & 9.42 & 183.5 & -84.47 & 2.46 & 2.46 & 1.21 & 11.10 & -0.17 \\
\hline 0.9 & 31.30 & 11.19 & 10.11 & 181.1 & -90.20 & 2.52 & 2.83 & 1.38 & 11.64 & -0.08 \\
\hline
\end{tabular}

